TRANSACTIONS OF THE

AMERICAN MATHEMATICAL SOCIETY

Volume 355, Number 3, Pages 1109-1133

S 0002-9947(02)03176-8

Article electronically published on October 25, 2002

\title{
LIMITS OF INTERPOLATORY PROCESSES
}

\author{
W. R. MADYCH
}

Abstract. Given $N$ distinct real numbers $\nu_{1}, \ldots, \nu_{N}$ and a positive approximation of the identity $\phi_{\epsilon}$, which converges weakly to the Dirac delta measure as $\epsilon$ goes to zero, we investigate the polynomials $P_{\epsilon}(x)=\sum c_{\epsilon, j} e^{-i \nu_{j} x}$ which solve the interpolation problem

$$
\int P_{\epsilon}(x) e^{i \nu_{k} x} \phi_{\epsilon}(x) d x=f_{\epsilon, k}, \quad k=1, \ldots, N,
$$

with prescribed data $f_{\epsilon, 1}, \ldots, f_{\epsilon, N}$. More specifically, we are interested in the behavior of $P_{\epsilon}(x)$ when the data is of the form $f_{\epsilon, k}=\int f(x) e^{i \nu_{k} x} \phi_{\epsilon}(x) d x$ for some prescribed function $f$. One of our results asserts that if $f$ is sufficiently nice and $\phi_{\epsilon}$ has sufficiently well-behaved moments, then $P_{\epsilon}$ converges to a limit $P$ which can be completely characterized. As an application we identify the limits of certain fundamental interpolatory splines whose knot set is $\mathbb{Z} \backslash \mathcal{N}$, where $\mathcal{N}$ is an arbitrary finite subset of the integer lattice $\mathbb{Z}$, as their degree goes to infinity.

1.

Suppose $\phi_{\epsilon}$ is a non-negative approximation of the identity. That is, $\epsilon$ is a positive, continuous or discrete, parameter with 0 as accumulation point and $\phi_{\epsilon}$ is a non-negative integrable function on the real line $\mathbb{R}$ with the properties (i) for all values of the parameter $\epsilon$,

$$
\int_{-\infty}^{\infty} \phi_{\epsilon}(x) d x=1
$$

and (ii) for every positive $\delta$,

$$
\lim _{\epsilon \rightarrow 0} \int_{|x|>\delta} \phi_{\epsilon}(x) d x=0 .
$$

The basic properties of such approximations of the identity $\phi_{\epsilon}$ are well known and are recorded in various texts on mathematical analysis, for example, [7]. In particular, if $f$ is a bounded measurable function which is continuous at the origin, then

$$
\lim _{\epsilon \rightarrow 0} \int_{-\infty}^{\infty} f(x) \phi_{\epsilon}(x) d x=f(0) .
$$

Given $N$ distinct real numbers $\nu_{1}, \ldots, \nu_{N}$, any point $\left(f_{1}, \ldots, f_{N}\right)$ in complex Euclidean $N$-space $\mathbb{C}^{N}$, and any fixed value $\epsilon$ of the parameter, there is a unique

Received by the editors April 11, 2002.

2000 Mathematics Subject Classification. Primary 41A05, 41A15.

(C)2002 American Mathematical Society 
polynomial

$$
P(x)=\sum_{j=1}^{N} c_{j} e^{-i \nu_{j} x}
$$

which satisfies

$$
\int_{-\infty}^{\infty} P(x) e^{i \nu_{k} x} \phi_{\epsilon}(x) d x=f_{k} \text { for } k=1, \ldots, N .
$$

The reason why $P(x)$ is uniquely defined is due to the fact that (2) is equivalent to the system of equations

$$
\sum_{j=1}^{N} a_{k, j} c_{j}=f_{k}, \quad k=1, \ldots, N,
$$

where the matrix of coefficients

$$
a_{k, j}=\int_{-\infty}^{\infty} e^{i\left(\nu_{k}-\nu_{l}\right) x} \phi_{\epsilon}(x) d x
$$

is strictly positive definite, as can be seen from

$$
\sum_{j, k} a_{k, j} c_{j} \bar{c}_{k}=\int_{-\infty}^{\infty}\left|\sum_{j=1}^{N} c_{j} e^{-i \nu_{j} x}\right|^{2} \phi_{\epsilon}(x) d x>0 .
$$

Consider the case when the data $\left(f_{1}, \ldots, f_{N}\right)$ is of the form

$$
f_{k}=\int_{-\infty}^{\infty} f(x) e^{i \nu_{k} x} \phi_{\epsilon}(x) d x
$$

where $f(x)$ is a function in $L^{\infty}$ which is continuous at 0 . The resulting polynomial $P$ depends on the function $f$ and $\phi_{\epsilon}$. To emphasize this dependence we denote it by $P_{f, \epsilon}$. That is, $P_{f, \epsilon}(x)$ is the unique polynomial of the form (1) which satisfies

$$
\int_{-\infty}^{\infty} P_{f, \epsilon}(x) e^{i \nu_{k} x} \phi_{\epsilon}(x) d x=\int_{-\infty}^{\infty} f(x) e^{i \nu_{k} x} \phi_{\epsilon}(x) d x, \quad k=1, \ldots, N .
$$

We are interested in the behavior of $P_{f, \epsilon}$ as the parameter $\epsilon$ goes to 0 .

In the case $N=1$ this behavior is transparent:

$$
P_{f, \epsilon}(x)=\left\{\int_{-\infty}^{\infty} f(y) e^{i \nu_{1} y} \phi_{\epsilon}(y) d y\right\} e^{-i \nu_{1} x},
$$

which, because $f$ is bounded and continous at 0 , converges to $f(0) e^{-i \nu_{1} x}$ as $\epsilon$ goes to zero.

In the case $N>1$ the situation is more interesting. When $\epsilon$ goes to zero the system of equations (3) for the coefficients $c_{j}$ of the polynomial $P_{f, \epsilon}$ reduces to

$$
\sum_{j=1}^{n} c_{j}=f(0) \text {. }
$$

That is, when $\epsilon$ goes to 0 the matrix of coefficients (4) converges to $a_{k, j}=1$ for every value of the index $(k, j)$ resulting in a non-invertible matrix of rank 1 . The behavior of $P_{f, \epsilon}$ is not so evident without further analysis.

In what follows we present some details of such an analysis. The impatient reader can turn to the statement of Theorem 7 in Section 6 for a succinct summary of one of the central conclusions. 
This investigation was motivated by questions arising from the study of the behavior of the family of splines of even order with knot set $\mathbb{Z} \backslash \mathcal{N}$, where $\mathcal{N}$ is a finite subset of the integer lattice $\mathbb{Z}$, which interpolate the data $\{(n, f(n))\}_{n \in \mathbb{Z} \backslash \mathcal{N}}$ with fixed ordinates $\{f(n)\}_{n \in \mathbb{Z} \backslash \mathcal{N}}$. In the case $\mathcal{N}=\emptyset$ such families were first studied by I. Schoenberg [6], who showed that if the data is sufficiently well behaved, such splines converge as the order goes to infinity. As an application we include a result concerning the convergence of such families of interpolants when $\mathcal{N} \neq \emptyset$. See Theorem 8 in Section 7.

It should be mentioned that, following Schoenberg [6], such convergence, with the set of knots taken to be a lattice, has been considered by many authors, including [2], where a general scenario is studied. In [5] such convergence has been investigated with the set of knots taken from a wider class of numerical sequences, namely the class of complete interpolating sequences for the classical Paley-Wiener space. The present application may be viewed as a step toward extending such results still further, since $\mathbb{Z} \backslash \mathcal{N}$ is not a complete interpolating sequence in the classical sense unless $\mathcal{N}=\emptyset$.

The contents of this article are briefly outlined below.

In Section 2 we collect several properties and examples of approximations of the identity which will be useful in what follows.

In Section 3 we outline some calculations in the case $N=2$ and record several types of results which depend both on the data $f$ and the nature of $\phi_{\epsilon}$.

Section 4 is devoted to observations concerning iterative formulas involving $N$, the number of frequencies, for the trigonometric polynomials $P_{f, \epsilon}(x)$ and their coefficients.

In Section 5 we record several identities for certain determinants which are useful in the computations involved in the case of general $N$. Some of these results may be of independent interest.

Section 6 contains our main results concerning the behavior of $P_{f, \epsilon}(x)$ as $\epsilon \rightarrow 0$. These results involve restrictions on $\phi_{\epsilon}$ which are fairly fundamental and enjoyed by wide classes of approximations of the identity.

In Section 7 we consider classical piecewise polynomial splines of even order with knots on the set $\mathbb{Z} \backslash \mathcal{N}$, where $\mathcal{N}$ is a finite subset of the integer lattice $\mathbb{Z}$, and study the behavior of the fundamental interpolants as their degree goes to infinity.

Section 8 is devoted to showing that the restrictions imposed on $\phi_{\epsilon}$ in Section 6 are enjoyed by a class of approximations of the identity which includes the case that arises in Section 7.

2.

We already noted that if the approximation of the identity $\phi_{\epsilon}$ is non-negative, then the $N \times N$ matrix $A=\left(a_{k, l}\right)$ with coefficients

$$
a_{k, l}=\int_{-\infty}^{\infty} e^{i\left(\nu_{k}-\nu_{l}\right) x} \phi_{\epsilon}(x) d x
$$

where the real numbers $\nu_{1}, \ldots, \nu_{N}$ are distinct, is strictly positive definite. Here we also note that if, in addition, the moments

$$
m_{k}(\epsilon)=\int_{-\infty}^{\infty} x^{k} \phi_{\epsilon}(x) d x
$$


are finite for $k=0,1, \ldots, 2(N-1)$, then the $N \times N$ matrix $\mathbf{M}=\left(M_{k, l}\right)$ with coefficients $M_{k, l}=m_{k+l}(\epsilon)$ is also strictly positive definite, because

$$
\sum_{k, l} M_{k, l} c_{k} \bar{c}_{l}=\int_{-\infty}^{\infty}\left|\sum_{k=1}^{N} c_{k} x^{k}\right|^{2} \phi_{\epsilon}(x) d x>0 .
$$

In particular, this implies that the determinant $\operatorname{det} \mathbf{M}$ is positive.

Consider an approximation of the identity of the form

$$
\phi_{\epsilon}(x)=\frac{1}{\epsilon} \phi\left(\frac{x}{\epsilon}\right)
$$

where $\phi$ is a non-negative integrable function, $\int_{-\infty}^{\infty} \phi(x) d x=1$, and $\epsilon>0$. In this case,

$$
m_{k}(\epsilon)=\epsilon^{k} m_{k}(1),
$$

so that if $m_{k}(1)$ is finite for $k=0,1, \ldots, 2(N-1)$, then the determinant of the $N \times N$ matrix $\mathbf{M}=\left(m_{k+l}\right)$ is simply a constant multiple of $\epsilon^{N(N-1)}$, namely

$$
\operatorname{det} \mathbf{M}=c \epsilon^{N(N-1)}
$$

where $c=\operatorname{det}\left(m_{k+l}(1)\right)$ is a positive constant. Identities (9) and (10) imply that in this case,

$$
\operatorname{det} \mathbf{M}=c\left\{m_{2(N-1)}(\epsilon)\right\}^{N / 2} .
$$

where $c=\operatorname{det}\left(m_{k+l}(1)\right) /\left\{m_{2(N-1)}(1)\right\}^{N / 2}$.

Next observe that if $w(x)$ is a bounded continuous function such that $w(0)=0$, then

$$
\int_{-\infty}^{\infty} w(x) x^{2(N-1)} \phi_{\epsilon}(x) d x=\epsilon^{2(N-1)} \int_{-\infty}^{\infty} w(\epsilon x) x^{2(N-1)} \phi(x) d x,
$$

and, in view of the dominated convergence theorem,

$$
\lim _{\epsilon \rightarrow 0} \int_{-\infty}^{\infty} w(\epsilon x) x^{2(N-1)} \phi(x) d x=0 .
$$

Hence, if $\phi_{\epsilon}$ is a non-negative approximation of the identity of the form (8), $m_{2(N-1)}(1)$ is finite, and $w(x)$ is a bounded continuous function such that $w(0)=0$, then

$$
\lim _{\epsilon \rightarrow 0} \frac{\int_{-\infty}^{\infty} w(x) x^{2(N-1)} \phi_{\epsilon}(x) d x}{\int_{-\infty}^{\infty} x^{2(N-1)} \phi_{\epsilon}(x) d x}=0 .
$$

Many important examples of approximations of the identity $\phi_{\epsilon}$ are either explicitly of the form (8), such as the Poisson and heat kernels for the upper half-space, or in many respects behave as though they are, such as certain periodic summability kernels including those of Féjer and de la Vallée Poussin. A noteworthy property of such approximations of the identity is that, roughly speaking, the rate of convergence of $\int_{-\infty}^{\infty} f(x) \phi_{\epsilon}(x) d x$ to $f(0)$ is essentially determined by the smoothness of $f$ in a neighborhood of 0 .

Details concerning approximations of the identity of type (8), including the kernels mentioned above, can be found in [7] and many other texts on mathematical analysis. A source for details on periodic summability kernels is [8]. 
Examples of "pathological" approximations of the identity whose behavior is somewhat transparent include the following:

$$
\phi_{\epsilon}(x)=\frac{1-\sigma(\epsilon)}{\epsilon} \phi(x / \epsilon)+\sigma(\epsilon) \theta(x)
$$

where both $\phi$ and $\theta$ are integrable functions such that

$$
\int_{-\infty}^{\infty} \phi(x) d x=\int_{-\infty}^{\infty} \theta(x) d x=1
$$

$\sigma(\epsilon)$ is an increasing positive function of $\epsilon, \epsilon>0$, and

$$
\lim _{\epsilon \rightarrow 0} \sigma(\epsilon)=0 .
$$

For examples of the form (13) the rate at which $\sigma(\epsilon)$ goes to zero can play a very significant role in determining the rate at which $\int_{-\infty}^{\infty} f(x) \phi_{\epsilon}(x) d x$ converges to $f(0)$, as can be easily seen from

$$
\int_{-\infty}^{\infty} f(x) \phi_{\epsilon}(x) d x=f(0)+\{1-\sigma(\epsilon)\} \int_{-\infty}^{\infty} w(\epsilon x) \phi(x) d x+\sigma(\epsilon) \int_{-\infty}^{\infty} w(x) \theta(x) d x,
$$

where $w(x)=f(x)-f(0)$.

3.

We begin our investigation of $P_{f, \epsilon}(x)$ with the case $N=2$. In this case an explicit formula for the coefficients of the polynomial

$$
P_{f, \epsilon}(x)=c_{\epsilon, 1} e^{-i \nu_{1} x}+c_{\epsilon, 2} e^{-i \nu_{2} x}
$$

which satisfies

$$
\int_{-\infty}^{\infty} P_{f, \epsilon}(x) e^{i \nu_{k} x} \phi_{\epsilon}(x) d x=\int_{-\infty}^{\infty} f(x) e^{i \nu_{k} x} \phi_{\epsilon}(x) d x \text { for } k=1,2
$$

can be obtained via Cramer's rule. This results in

$$
c_{\epsilon, 1}=\frac{G\left(\nu_{1}\right)-G\left(\nu_{2}\right) \Phi\left(\nu_{1}-\nu_{2}\right)}{1-\Phi\left(\nu_{1}-\nu_{1}\right) \Phi\left(\nu_{2}-\nu_{1}\right)}
$$

where

$$
G(z)=\int_{-\infty}^{\infty} f(x) e^{i z x} \phi_{\epsilon}(x) d x
$$

and

$$
\Phi(z)=\int_{-\infty}^{\infty} e^{i z x} \phi_{\epsilon}(x) d x .
$$

A similar expression is valid for $c_{\epsilon, 2}$. For efficiency of notation we have chosen not to explicitly express the dependence of $G$ and $\Phi$ on $\epsilon$.

To see what can happen to $c_{\epsilon, 1}$ as $\epsilon$ goes to zero, assume that the moments

$$
m_{k}(\epsilon)=\int_{-\infty}^{\infty} x^{k} \phi_{\epsilon}(x) d x
$$

are finite for $k=1$ and 2. For simplicity assume that $\phi_{\epsilon}$ is a nice approximation of the identity which behaves like those of the form (8). More specifically, assume there are positive constants $r_{0} \leq r_{1}$ such that

$$
0<r_{0} \leq \frac{m_{2}(\epsilon)-\left(m_{1}(\epsilon)\right)^{2}}{m_{2}(\epsilon)} \leq r_{1}<\infty
$$


for all positive values of the parameter $\epsilon$ and that

$$
\lim _{\epsilon \rightarrow 0} \frac{\int_{-\infty}^{\infty} \omega(x) x^{2} \phi_{\epsilon}(x) d x}{m_{2}(\epsilon)}=0
$$

whenever $\omega$ is a bounded continuous function with $\omega(0)=0$. Also assume that $f$ is a bounded function which is continuously differentiable in a neighborhood of the origin.

Write

where

$$
e^{i z x}=1+i z x+(i z)^{2} \frac{x^{2}}{2}+w_{z}(x) \frac{x^{2}}{2}
$$

$$
w_{z}(x)=(i z)^{2}\left\{\cos z x_{1}-1+i \sin z x_{2}\right\}
$$

and $x_{1}$ and $x_{2}$ are appropriately chosen points between 0 and $x$. Hence

$$
\Phi(z)=1+i z m_{1}(\epsilon)+(i z)^{2} \frac{m_{2}(\epsilon)}{2}+o\left(m_{2}(\epsilon)\right)
$$

where, as is customary, the notation $C=o\left(m_{2}(\epsilon)\right)$ means that

$$
\lim _{\epsilon \rightarrow 0} \frac{C}{m_{2}(\epsilon)}=0 \text {. }
$$

Thus

$$
\Phi(z) \Phi(-z)=1+(i z)^{2}\left\{m_{2}-m_{1}^{2}\right\}+o\left(m_{2}\right)
$$

where, for simplicity, the explicit dependence of $m_{1}$ and $m_{2}$ on $\epsilon$ has been dropped from the notation.

Similarly,

$$
\begin{aligned}
G(z)= & \int_{-\infty}^{\infty} f(x) \phi_{\epsilon}(x) d x+f(0)\left\{i z m_{1}+(i z)^{2} \frac{m_{2}}{2}\right\} \\
& +i z \int_{-\infty}^{\infty} \omega_{f}(x) x \phi_{\epsilon}(x) d x+o\left(m_{2}\right)
\end{aligned}
$$

where $\omega_{f}(x)=f(x)-f(0)$. Combining (19) and (21) and, for simplicity, setting $a=\nu_{1}$ and $b=\nu_{2}$ results in

$$
\begin{aligned}
G(a)- & G(b) \Phi(a-b) \\
= & i b(i a-i b) f(0)\left\{m_{2}-m_{1}^{2}\right\} \\
& +(i a-i b) \int_{-\infty}^{\infty} \omega_{f}(x)\left\{x-m_{1}\right\} \phi_{\epsilon}(x) d x+o\left(m_{2}\right) .
\end{aligned}
$$

Writing, for appropriately chosen points $x_{1}$ and $x_{2}$ between 0 and $x$,

$$
\omega_{f}(x)=\left\{\operatorname{Re} f^{\prime}\left(x_{1}\right)+i \operatorname{Im} f^{\prime}\left(x_{2}\right)\right\} x=\left\{f^{\prime}(0)+\omega_{f^{\prime}}(x)\right\} x,
$$

where $\omega_{f^{\prime}}(x)=\operatorname{Re}\left(f^{\prime}\left(x_{1}\right)-f^{\prime}(0)\right)+i \operatorname{Im}\left(f^{\prime}\left(x_{2}\right)-f^{\prime}(0)\right)$, yields

$$
G(a)-G(b) \Phi(a-b)=\left\{i b f(0)+f^{\prime}(0)\right\}(i a-i b)\left\{m_{2}-m_{1}^{2}\right\}+o\left(m_{2}\right) .
$$

In the last calculation we also used the facts that

$$
\begin{gathered}
\int_{-\infty}^{\infty} \omega(x) x \phi_{\epsilon}(x) d x \leq\left\{\int_{-\infty}^{\infty} \omega(x)^{2} \phi_{\epsilon}(x) d x\right\}^{1 / 2}\left\{\int_{-\infty}^{\infty} x^{2} \phi_{\epsilon}(x) d x\right\}^{1 / 2}, \\
\int_{-\infty}^{\infty} x \phi_{\epsilon}(x) d x \leq\left\{\int_{-\infty}^{\infty} x^{2} \phi_{\epsilon}(x) d x\right\}^{1 / 2}
\end{gathered}
$$


and

$$
m_{1} \int_{-\infty}^{\infty} \omega(x) x \phi_{\epsilon}(x) d x \leq m_{2}\left\{\int_{-\infty}^{\infty} \omega(x)^{2} \phi_{\epsilon}(x) d x\right\}^{1 / 2}=o\left(m_{2}\right) .
$$

Identities (20) and (23) imply

$$
\frac{G(a)-G(b) \Phi(a-b)}{1-\Phi(a-b) \Phi(b-a)}=\frac{\left\{i b f(0)+f^{\prime}(0)\right\}(i a-i b)\left\{m_{2}-m_{1}^{2}\right\}+o\left(m_{2}\right)}{-(i a-i b)^{2}\left\{m_{2}-m_{1}^{2}\right\}+o\left(m_{2}\right)},
$$

so that, in view of relation (17) together with the substitutions $a=\nu_{1}$ and $b=\nu_{2}$, we may write

$$
\lim _{\epsilon \rightarrow 0} c_{\epsilon, 1}=c_{1}=\frac{f^{\prime}(0)+i \nu_{2} f(0)}{i \nu_{2}-i \nu_{1}} .
$$

A similar calculation shows that

$$
\lim _{\epsilon \rightarrow 0} c_{\epsilon, 2}=c_{2}=\frac{f^{\prime}(0)+i \nu_{1} f(0)}{i \nu_{1}-i \nu_{2}} .
$$

The limits $c_{1}$ and $c_{2}$ in (24) and (25) respectively, it can be verified, are the coefficients of the polynomial

$$
P_{f}(x)=c_{1} e^{-i \nu_{1} x}+c_{2} e^{-i \nu_{2} x},
$$

which satisfies

$$
P_{f}(0)=f(0) \text { and } P_{f}^{\prime}(0)=f^{\prime}(0) .
$$

We summarize the results of the above calculations as follows.

Theorem 1. Suppose $\phi_{\epsilon}$ is an approximation of the identity which enjoys properties (17) and (18), $f$ is a bounded function which is continuously differentiable in a neighborhood of the origin, and $P_{f, \epsilon}$ is the polynomial (14) which satisfies (15). Then

$$
\lim _{\epsilon \rightarrow 0} P_{f, \epsilon}(x)=P_{f}(x)
$$

where $P_{f}(x)$ is the unique polynomial (26) which enjoys property (27).

It should be clear from (22) that if $\phi_{\epsilon}$ satisfies the hypotheses of the theorem but $f$ fails to be continuously differentiable in a neighborhood of the origin, then $P_{f, \epsilon}$ may fail to converge. For example, take $\phi_{\epsilon}(x)=\phi(x / \epsilon) / \epsilon$ where $\phi(x)=1$ when $|x| \leq 1 / 2$ and 0 otherwise. Then, if $f(x)=|x|^{\alpha}\{x /|x|\}$ in a neighborhood of the origin, using (22), for sufficiently small $\epsilon$ we get

$$
G(a)-G(b) \Phi(a-b)=\frac{i a-i b}{\epsilon} \int_{-\epsilon / 2}^{\epsilon / 2}|x|^{\alpha+1} d x+o\left(\epsilon^{2}\right),
$$

so that

$$
c_{\epsilon, 1}=\frac{\epsilon^{\alpha-1} /(\alpha+2) 2^{\alpha}+o\left(\epsilon^{2}\right) / \epsilon^{2}}{(i b-i a) / 6+o\left(\epsilon^{2}\right) / \epsilon^{2}}=0\left(\epsilon^{\alpha-1}\right),
$$

which "blows up" as $\epsilon \rightarrow 0$ if $0<\alpha<1$.

On the other hand, if $\phi_{\epsilon}$ does not satisfy (17) and/or (18), then other interesting phenomena can occur. For example, consider $\phi_{\epsilon}$ of the form (13) where $\sigma(\epsilon)$ converges to zero "slowly" as $\epsilon$ goes to 0 . Specifically, since we are interested in $P_{f, \epsilon}$ with $f$ continuous at the origin, assume that

$$
\max (\epsilon, \omega(\epsilon))=o(\sigma(\epsilon)) \text { as } \epsilon \rightarrow 0,
$$


where $\omega(\epsilon)=\sup _{|x| \leq \epsilon}|f(x)-f(0)|$. Also assume that

$$
\int_{-\infty}^{\infty}|x| \phi(x) d x<\infty
$$

Using the fact that (29) implies that $\int_{-\infty}^{\infty}|x| \phi(x / \epsilon) / \epsilon d x=O(\epsilon)$ and $\int_{-\infty}^{\infty} \omega(|x|) \phi(x / \epsilon) / \epsilon d x=O(\max (\epsilon, \omega(\epsilon)))$, we may write

$$
\Phi(z) \Phi(-z)=1+\sigma(\epsilon) \int_{-\infty}^{\infty}\left\{w_{z}(x)+w_{-z}(x)\right\} \theta(x) d x+o(\sigma(\epsilon))
$$

and

$$
G(a)-G(b) \Phi(a-b)=\sigma(\epsilon) \int_{-\infty}^{\infty} w_{a-b}(x)\left\{e^{i b x} f(x)-f(0)\right\} \theta(x) d x+o(\sigma(\epsilon)),
$$

where $w_{z}(x)=e^{i z x}-1$. The last two identities imply that

$$
\lim _{\epsilon \rightarrow 0} c_{\epsilon, 1}=c_{1}=\frac{\int_{-\infty}^{\infty}\left\{e^{i\left(\nu_{1}-\nu_{2}\right) x}-1\right\}\left\{e^{i \nu_{2} x} f(x)-f(0)\right\} \theta(x) d x}{2 \int_{-\infty}^{\infty}\left\{1-\cos \left(\left(\nu_{1}-\nu_{2}\right) x\right)\right\} \theta(x) d x} .
$$

The last ratio is finite whenever the denominator is not 0 , which is necessarily the case if, for example, $\theta$ is non-negative. A similar formula holds for $c_{2}=\lim _{\epsilon \rightarrow 0} c_{\epsilon, 2}$ : simply interchange $\nu_{1}$ and $\nu_{2}$ in the right-hand side of (30). Let $P$ be the corresponding trigonometric polynomial, namely

$$
P(x)=c_{1} e^{-i \nu_{1} x}+c_{2} e^{-i \nu_{2} x} .
$$

Then the results of the above calculation may be summarized as follows.

Theorem 2. Suppose the approximation of the identity $\phi_{\epsilon}$ is of the form (13), $\phi$ satisfies (29), and $\sigma(\epsilon)$ satisfies $\lim _{\epsilon \rightarrow 0} \epsilon / \sigma(\epsilon)=0$. If $P_{f, \epsilon}(x)$ is the polynomial (14) which satisfies (15), then

$$
\lim _{\epsilon \rightarrow 0} P_{f, \epsilon}(x)=P(x),
$$

where $P(x)$ is the trigonometric polynomial (31) whose coefficients are determined by (30), whenever $f$ is a bounded function which is continuous at 0 and whose modulus of continuity at zero, $\omega(\epsilon)=\sup _{|x| \leq \epsilon}|f(x)-f(0)|$, satifies $\omega(\epsilon)=o(\sigma(\epsilon))$.

Remark 3.1. In what follows we will use the mean value theorem and its variants in various calculations. For example, for a complex-valued function $f$ of the real variable $x$ we have

$$
f(x)-f(0)=\left\{\operatorname{Re} f^{\prime}\left(x_{1}\right)+i \operatorname{Im} f^{\prime}\left(x_{2}\right)\right\} x
$$

for appropriately chosen $x_{1}$ and $x_{2}$ between 0 and $x$. As is evident from the outline of the calculation preceding the statement of Theorem 1 (see the derivations of identities (19) and (23)), the use of such explicit notation is rather cumbersome. For simplicity, in what follows we will express relations such as (32) above as

$$
f(x)-f(0)=f^{\prime}(\tilde{x}) x
$$

with the understanding that the real and imaginary parts must be treated separately, namely, $f^{\prime}(\tilde{x})=\operatorname{Re} f^{\prime}\left(x_{1}\right)+i \operatorname{Im} f^{\prime}\left(x_{2}\right)$. 
4.

In view of the observations made in the previous section, it is natural to conjecture that if $f$ is sufficiently smooth in a neighborhood of the origin and $\phi_{\epsilon}$ is sufficiently well behaved, then the polynomials $P_{f, \epsilon}$ converge as $\epsilon$ goes to 0 in the case when more that two frequencies are involved. It also seems reasonable, particularly in view of Theorem 1, that an argument involving induction on $N$, the number of frequencies $\nu_{1}, \ldots, \nu_{N}$, may come into play.

With this in mind we temporarily modify our notation to take into account the dependence of $P_{f, \epsilon}$ on the number of frequencies involved. Thus, (i) let $\nu_{1}, \nu_{2}, \ldots$ be a sequence of distinct real numbers; (ii) for $N=1,2, \ldots$ define $P_{f, \epsilon, N}(x)$ to be the trigonometric polynomial (11) with frequencies $\nu_{1}, \ldots, \nu_{N}$; (iii) let $\mathbf{A}_{N}=\left(a_{k, l}\right)$ be the $N \times N$ matrix with coefficients

$$
a_{k, l}=\Phi\left(\nu_{k}-\nu_{l}\right)=\int_{-\infty}^{\infty} e^{i\left(\nu_{k}-\nu_{l}\right) x} \phi_{\epsilon}(x) d x, \quad k, l=1, \ldots, N
$$

(iv) let $\mathbf{c}_{N}$ be the column vector whose terms $\left(c_{1}, \ldots, c_{N}\right)$ are the coefficients of $P_{f, \epsilon, N}(x) ;(\mathrm{v})$ let $\mathbf{f}_{N}$ be the column vector whose terms $\left(f_{1}, \ldots, f_{N}\right)$ are given by $f_{k}=\int_{-\infty}^{\infty} f(x) e^{i \nu_{k} x} \phi_{\epsilon}(x) d x$; and (vi) set $\lambda_{z}(x)=e^{-i z x}$. Then

$$
\mathbf{A}_{N} \mathbf{c}_{N}=\mathbf{f}_{N}
$$

and

$$
\mathbf{A}_{N+1} \mathbf{c}_{N+1}=\left(\begin{array}{cc}
\mathbf{A}_{N} & \mathbf{a}_{N} \\
\mathbf{a}_{N}^{*} & 1
\end{array}\right)\left(\begin{array}{c}
\mathbf{c} \\
c_{N+1}
\end{array}\right)=\left(\begin{array}{c}
\mathbf{f}_{N} \\
f_{N+1}
\end{array}\right)
$$

where $\mathbf{a}_{N}=\mathbf{f}_{N}$ with the particular choice $f(x)=\lambda_{\nu_{N+1}}(x)$, $\mathbf{a}_{N}^{*}$ is the conjugate transpose of $\mathbf{a}_{N}$, and $\mathbf{c}$ is the column vector consisting of the first $N$ components of $\mathbf{c}_{N+1}$.

To better understand the relationship between these quantities, re-express the last displayed identity as

$$
\begin{aligned}
\mathbf{A}_{N} \mathbf{c}+c_{N+1} \mathbf{a}_{N} & =\mathbf{f}_{N}, \\
\mathbf{a}_{N}^{*} \mathbf{c}+c_{N+1} & =f_{N+1},
\end{aligned}
$$

which can be easily solved for $\mathbf{c}$ and $c_{N+1}$ to yield

$$
c_{N+1}=\frac{f_{N+1}-\mathbf{a}_{N}^{*} \mathbf{A}_{N}^{-1} \mathbf{f}_{N}}{1-\mathbf{a}_{N}^{*} \mathbf{A}_{N}^{-1} \mathbf{a}_{N}}
$$

and

$$
\mathbf{c}=\mathbf{A}_{N}^{-1} \mathbf{f}_{N}-\left\{\frac{f_{N+1}-\mathbf{a}_{N}^{*} \mathbf{A}_{N}^{-1} \mathbf{f}_{N}}{1-\mathbf{a}_{N}^{*} \mathbf{A}_{N}^{-1} \mathbf{a}_{N}}\right\} \mathbf{A}_{N}^{-1} \mathbf{a}_{N} .
$$

Since $\mathbf{A}_{N}^{-1} \mathbf{f}_{N}$ and $\mathbf{A}_{N}^{-1} \mathbf{a}_{N}$ are the coefficients of $P_{f, \epsilon, N}(x)$ and $P_{\lambda_{z}, \epsilon, N}(x)$ respectively with $z=\nu_{N+1}$, formulas (34) and (35) may be viewed as a recursive relationship for the coefficents of $P_{f, \epsilon, N+1}(x)$ in terms of those of $P_{f, \epsilon, N}(x)$. Indeed, using the notation

$$
\langle f, g\rangle_{\epsilon}=\int_{-\infty}^{\infty} f(x) \overline{g(x)} \phi_{\epsilon}(x) d x
$$

formulas (34) and (35) may be expressed as

$$
P_{f, \epsilon, N+1}(x)=P_{f, \epsilon, N}(x)+\frac{\left\langle f-P_{f, \epsilon, N}, \lambda_{z}\right\rangle_{\epsilon}}{\left\langle\lambda_{z}-P_{\lambda_{z}, \epsilon, N}, \lambda_{z}\right\rangle_{\epsilon}}\left\{\lambda_{z}(x)-P_{\lambda_{z}, \epsilon, N}(x)\right\}
$$


where $z=\nu_{N+1}$.

Now, if $P_{f, 0, N}(x)$ denotes the unique polynomial of the form (11) which satisfies $P^{(k)}(0)=f^{(k)}(0)$ for $k=0, \ldots, N-1$, then it is not difficult to see that

$$
P_{f, 0, N+1}(x)=P_{f, 0, N}(x)+\frac{f^{(N)}(0)-P_{f, 0, N}^{(N)}(0)}{\lambda_{z}^{(N)}(0)-P_{\lambda_{z}, 0, N}^{(N)}(0)}\left\{\lambda_{z}(x)-P_{\lambda_{z}, 0, N}(x)\right\},
$$

where $z=\nu_{N+1}$. Formulas (36) and (37) seem to justify an inductive type argument. Unfortunately we were unable to make efficient use of such recursive type relationships. For example, such relationships do not readily yield a clue as to the "correct" set of restrictions needed on $\phi_{\epsilon}$ in order to obtain an appropriate result. We found a more direct approach to be more efficient. Nevertheless, these identities are useful, if for nothing more than providing a hint as to the importance of the role played by $\lambda_{z}(x)$ and $P_{\lambda_{z}, \epsilon, N}(x)$, which in turn provided the basis to our direct approach.

5.

In what follows all indices such as $j, k$, and $l$ take on the values $1, \ldots, N$ unless specifically indicated otherwise. The values $\nu_{1}, \ldots, \nu_{N}$ are distinct real numbers. $P_{f, \epsilon}(x), \epsilon>0$, and $P_{f, 0}(x)$ are trigonometric polynomials of type (11). $P_{f, \epsilon}(x)$ is the unique polynomial which satisfies (5). $P_{f, 0}(x)$ is defined only if $f$ is sufficiently smooth, in which case it is the unique polynomial which satisfies $P_{f, 0}^{(k)}(0)=f^{(k)}(0)$, $k=0, \ldots, N-1$. Also, let

$$
\Phi(z)=\int_{-\infty}^{\infty} e^{i z x} \phi_{\epsilon}(x) d x .
$$

In the preceding section we noted the importance of the role played by $P_{\lambda_{z}, \epsilon}(x)$ and $P_{\lambda_{z}, 0}(x)$. The coefficients of these polynomials are given by

$$
c_{\epsilon, j}=\frac{\operatorname{det}\left(\Phi\left(u_{k}-\nu_{l}\right)\right)}{\operatorname{det}\left(\Phi\left(\nu_{k}-\nu_{l}\right)\right)}
$$

and

$$
c_{0, j}=\frac{\mathcal{V}\left(-i u_{1}, \ldots-i u_{N}\right)}{\mathcal{V}\left(-i \nu_{1}, \ldots,-i \nu_{N}\right)}
$$

respectively, where $u_{k}=z$ if $k=j$ and $\nu_{k}$ otherwise. $\mathcal{V}\left(v_{1}, \ldots, v_{N}\right)$ is the Vandermonde determinant:

$$
\mathcal{V}\left(v_{1}, \ldots, v_{N}\right)=\operatorname{det}\left(\left(v_{l}\right)^{k-1}\right)=\prod_{k>l}\left(v_{k}-v_{l}\right) .
$$

We would like to ensure that under suitable restrictions on $\phi_{\epsilon}$, the coefficients $c_{\epsilon, j}$ converge to $c_{0, j}$ as $\epsilon \rightarrow 0$.

To this end, consider the $N \times N$ matrix

$$
\left(\Phi\left(u_{k}-v_{l}\right)\right)=\left(\begin{array}{cccc}
\Phi\left(u_{1}-v_{1}\right) & \Phi\left(u_{1}-v_{2}\right) & \ldots & \Phi\left(u_{1}-v_{N}\right) \\
\Phi\left(u_{2}-v_{1}\right) & \ldots & & \\
\vdots & & & \\
\Phi\left(u_{N}-v_{1}\right) & \ldots & & \Phi\left(u_{N}-v_{N}\right)
\end{array}\right)
$$

parametrized by the $2 N$ variables $u_{1}, \ldots, u_{N}$ and $v_{1}, \ldots, v_{N}$ where $\Phi(z)$ is an analytic function of $z$. We can obtain a useful identity for the determinant of this 
matrix by observing that (i) if we fix $2 N-1$ of the variables, then this determinant is analytic in the remaining variable, and (ii) if $u_{k}=u_{l}$ for $k \neq l$, then this determinant must be 0 . For example, if we take the "remaining" variable to be $u_{1}$, then the determinant as an analytic function of $u_{1}$ has zeros $u_{2}, \ldots, u_{N}$. These observations allow us to conclude that

$$
\operatorname{det}\left(\Phi\left(u_{k}-u_{l}\right)\right)=\left\{\prod_{k>l}\left(u_{k}-u_{l}\right)\left(v_{k}-v_{l}\right)\right\} \Psi\left(u_{1}, \ldots, u_{N} ; v_{1}, \ldots, v_{N}\right)
$$

where, as indicated, $\Psi$ is a function of the $2 N$ variables $u_{1}, \ldots, u_{N}$ and $v_{1}, \ldots, v_{N}$. This, of course, is very encouraging, since it seems to indicate that, with appropriate cancellations, the ratio (38) looks pretty much like (39) if the factors involving $\Psi$ cooperate as $\epsilon \rightarrow 0$.

Investigation of the nature of $\Psi$ in formula (41) leads to consideration of certain divided differences of $\Phi(z)$. Because of the cumbersome nature of the expressions involved, it eventually becomes apparent that it may be more efficient to formulate the results in terms of the more general matrix $\left(F\left(u_{k}, v_{l}\right)\right)$ in lieu of $\left(\Phi\left(u_{k}-v_{l}\right)\right)$. The details are outlined in what follows.

We begin by reviewing some definitions and facts concerning divided differences which are useful in understanding the nature of $\Psi$ in identity (41).

The divided difference of $y_{1}, \ldots, y_{N}$ with respect to the distinct points $x_{1}, \ldots, x_{N}$ is denoted by

$$
\left[y_{1}, \ldots, y_{N} ; x_{1}, \ldots, x_{N}\right]
$$

and defined inductively by

$$
\left[y_{1} ; x_{1}\right]=y_{1}
$$

and

$$
\begin{aligned}
{\left[y_{1}, \ldots, y_{N+1} ; x_{1}, \ldots, x_{N+1}\right] } & \\
& =\frac{\left[y_{2}, \ldots, y_{N+1} ; x_{2}, \ldots, x_{N+1}\right]-\left[y_{1}, \ldots, y_{N} ; x_{1}, \ldots, x_{N}\right]}{x_{N+1}-x_{1}} .
\end{aligned}
$$

This particular notation, which is useful in the definitions below, follows that used in [4]; properties of divided differences are recorded in various texts on numerical analysis, for example [3]. Note that $\left[y_{1}, \ldots, y_{N} ; x_{1}, \ldots, x_{N}\right]$ is a divided difference of order $N-1$.

For a function $G(u)$ of one variable, the divided difference $G\left(\left[u_{1}, \ldots, u_{M}\right]\right)$ is defined in terms of (42) as

$$
G\left(\left[u_{1}, \ldots, u_{M}\right]\right)=\left[G\left(u_{1}\right), \ldots, G\left(u_{M}\right) ; u_{1}, \ldots, u_{M}\right] .
$$

For a function $F(u, v)$ of two variables we have

$$
F\left(\left[u_{1}, \ldots, u_{M}\right], v\right)=\left[F\left(u_{1}, v\right), \ldots, F\left(u_{M}, v\right) ; u_{1}, \ldots, u_{M}\right]
$$

and

$$
F\left(u,\left[v_{1}, \ldots, v_{N}\right]\right)=\left[F\left(u, v_{1}\right), \ldots, F\left(u, v_{N}\right) ; v_{1}, \ldots, v_{N}\right] .
$$

The divided difference $F\left(\left[u_{1}, \ldots, u_{M}\right],\left[v_{1}, \ldots, v_{N}\right]\right)$ can be defined in terms of the previous expressions and (42) by the formula

$$
\begin{aligned}
& F\left(\left[u_{1}, \ldots, u_{M}\right],\left[v_{1}, \ldots, v_{M}\right]\right) \\
& \quad=\left[F\left(u_{1},\left[v_{1}, \ldots, v_{N}\right]\right), \ldots, F\left(u_{M},\left[v_{1}, \ldots, v_{N}\right]\right) ; u_{1}, \ldots, u_{M}\right] .
\end{aligned}
$$


Note that $F\left(\left[u_{1}, \ldots, u_{M}\right],\left[v_{1}, \ldots, v_{N}\right]\right)$, which may be viewed as some sort of mixed divided difference of order $M-1+N-1$, is well defined because

$$
\begin{aligned}
& {\left[F\left(u_{1},\left[v_{1}, \ldots, v_{N}\right]\right), \ldots, F\left(u_{M},\left[v_{1}, \ldots, v_{N}\right]\right) ; u_{1}, \ldots, u_{M}\right]} \\
& =\left[F\left(\left[u_{1}, \ldots, u_{M}\right], v_{1}\right), \ldots, F\left(\left[u_{1}, \ldots, u_{M}\right], v_{N}\right) ; v_{1}, \ldots, v_{N}\right] .
\end{aligned}
$$

If $u_{1}, \ldots, u_{M}$ and $v_{1}, \ldots, v_{N}$ are real and $F$ is a sufficiently smooth real-valued function, then, for appropriately chosen $\tilde{u}$ and $\tilde{v}$ which are in the convex hull of $u_{1}, \ldots, u_{M}$ and $v_{1}, \ldots, v_{N}$ respectively, an iteration of a standard identity for divided differences, for examples see [3, Corollary 3.4.2], results in

$$
\begin{aligned}
F\left(\left[u_{1}, \ldots, u_{M}\right],\left[v_{1}, \ldots, v_{N}\right]\right) \\
\quad=\left.\frac{1}{(M-1) !}\left(\frac{\partial}{\partial u}\right)^{M-1}\left[F\left(u, v_{1}\right), \ldots, F\left(u, v_{N}\right) ; v_{1}, \ldots, v_{N}\right]\right|_{u=\tilde{u}} \\
\quad=\frac{1}{(M-1) !}\left[\left.\frac{\partial^{M-1} F\left(u, v_{1}\right)}{\partial u^{M-1}}\right|_{u=\tilde{u}}, \ldots,\left.\frac{\partial^{M-1} F\left(u, v_{N}\right)}{\partial u^{M-1}}\right|_{u=\tilde{u}} ; v_{1}, \ldots, v_{N}\right] \\
\quad=\left.\frac{1}{(N-1) !} \frac{1}{(M-1) !}\left(\frac{\partial}{\partial v}\right)^{N-1}\left(\frac{\partial}{\partial u}\right)^{M-1} F(u, v)\right|_{(u, v)=(\tilde{u}, \tilde{v})} .
\end{aligned}
$$

This may be summarized for future use as

$$
\begin{aligned}
& F\left(\left[u_{1}, \ldots, u_{M}\right],\left[v_{1}, \ldots, v_{N}\right]\right) \\
& \quad=\left.\frac{1}{(N-1) !} \frac{1}{(M-1) !}\left(\frac{\partial}{\partial v}\right)^{N-1}\left(\frac{\partial}{\partial u}\right)^{M-1} F(u, v)\right|_{(u, v)=(\tilde{u}, \tilde{v})} .
\end{aligned}
$$

For complex-valued $F$, identity (44) is valid for the real and complex part of $F$ with possibly different choices of $(\tilde{u}, \tilde{v})$ respectively. However, as indicated by Remark 3.1, we will retain the symbolic notation (44) for complex-valued $F$ with the understanding that possibly different choices of $(\tilde{u}, \tilde{v})$ may be necessary for the real and imaginary parts respectively.

Given a function $F(u, v)$ and collections $U=\left\{u_{1}, \ldots, u_{N}\right\}$ and $V=\left\{v_{1}, \ldots\right.$, $\left.v_{N}\right\}$, let $\left(F\left(u_{k}, v_{l}\right)\right)$ be the $N \times N$ matrix

$$
\left(F\left(u_{k}, v_{l}\right)\right)=\left(\begin{array}{llll}
F\left(u_{1}, v_{1}\right) & F\left(u_{1}, v_{2}\right) & \cdots & F\left(u_{1}, v_{N}\right) \\
F\left(u_{2}, v_{1}\right) & \cdots & & \\
\vdots & & & \\
F\left(u_{N}, v_{1}\right) & F\left(u_{N}, u_{2}\right) & \cdots & F\left(u_{N}, v_{N}\right)
\end{array}\right)
$$

and let $\left(F\left(\left[U_{k}\right],\left[V_{l}\right]\right)\right)$ be the $N \times N$ matrix consisting of divided differences

$$
\left(F\left(\left[U_{k}\right],\left[V_{l}\right]\right)\right)=\left(\begin{array}{llll}
F\left(\left[U_{1}\right],\left[V_{1}\right]\right) & F\left(\left[U_{1}\right],\left[V_{2}\right]\right) & \cdots & F\left(\left[U_{1}\right],\left[V_{N}\right]\right) \\
F\left(\left[U_{2}\right],\left[V_{1}\right]\right) & \cdots & & \\
\vdots & & & \\
F\left(\left[U_{N}\right],\left[V_{1}\right]\right) & F\left(\left[U_{N}\right],\left[V_{2}\right]\right) & \cdots & F\left(\left[U_{N}\right],\left[V_{N}\right]\right)
\end{array}\right)
$$

where $U_{k}=\left\{u_{1}, \ldots, u_{k}\right\}$ and $V_{k}=\left\{v_{1}, \ldots, v_{k}\right\}$ for $k=1, \ldots, N$. In other words,

$$
\left(F\left(\left[U_{k}\right],\left[V_{l}\right]\right)\right)=\left(F\left(\left[u_{1}, \ldots, u_{k}\right],\left[v_{1}, \ldots, v_{l}\right]\right)\right) .
$$




\section{Theorem 3.}

$$
\operatorname{det}\left(F\left(u_{k}, v_{l}\right)\right)=\left\{\prod_{k>l}\left(u_{k}-u_{l}\right)\left(v_{k}-v_{l}\right)\right\} \operatorname{det}\left(F\left(\left[U_{k}\right],\left[V_{l}\right]\right)\right) .
$$

Proof. First, for simplicity, write $\mathbf{F}=\left(F\left(u_{k}, v_{l}\right)\right)$. Next, subtract the first column of $\mathbf{F}$ from the succeeding $N-1$ columns of $\mathbf{F}$ and call the resulting matrix $\tilde{\mathbf{F}}$. Now multiply the $k$ th column of $\tilde{\mathbf{F}}$ by $\frac{1}{v_{k}-v_{1}}$ for $k=2, \ldots, N$ and call the result $\mathbf{F}^{[1]}$. Note that

$$
\operatorname{det} \mathbf{F}=\operatorname{det} \tilde{\mathbf{F}}
$$

and

$$
\tilde{\mathbf{F}}=\mathbf{F}^{[1]} \operatorname{diag}\left(1, v_{2}-v_{1}, \ldots, v_{N}-v_{1}\right)
$$

where $\operatorname{diag}\left(a_{1}, \ldots, a_{N}\right)$ denotes the $N \times N$ matrix with diagonal $\left(a_{1}, \ldots, a_{N}\right)$ and with zeros off the diagonal. The last two identities imply that

$$
\operatorname{det} \mathbf{F}=\left\{\prod_{k=2}^{N}\left(v_{k}-v_{1}\right)\right\} \operatorname{det} \mathbf{F}^{[1]}
$$

Next subtract the second column of $\mathbf{F}^{[1]}$ from each of the succeeding $N-2$ columns, call the result $\widetilde{\mathbf{F}}^{[1]}$, multiply the $k$ th column of $\widetilde{\mathbf{F}}^{[1]}$ by $\frac{1}{v_{k}-v_{2}}$ for $k=3, \ldots, N$, and call the final result $\mathbf{F}^{[2]}$. As above, note that

$$
\operatorname{det} \mathbf{F}^{[1]}=\operatorname{det} \widetilde{\mathbf{F}}^{[1]}
$$

and

$$
\widetilde{\mathbf{F}}^{[1]}=\mathbf{F}^{[2]} \operatorname{diag}\left(1,1, v_{3}-v_{2}, \ldots, v_{N}-v_{1}\right)
$$

so it is clear that

$$
\operatorname{det} \mathbf{F}^{[1]}=\left\{\prod_{k=3}^{N}\left(v_{k}-v_{2}\right)\right\} \operatorname{det} \mathbf{F}^{[2]} .
$$

If necessary, continue in this way to get $\mathbf{F}^{[3]}, \ldots, \mathbf{F}^{[N-1]}$. The $k$ th column of $\mathbf{F}^{[N-1]}$ consists of divided differences of order $k-1$ with respect to $v_{1}, \ldots, v_{k}$. The relations (47) and (48) may be extended to

$$
\operatorname{det} \mathbf{F}^{[n-1]}=\left\{\prod_{k=n+1}^{N}\left(v_{k}-v_{n}\right)\right\} \operatorname{det} \mathbf{F}^{[n]}
$$

for $n=1, \ldots, N-1$.

Next, apply the same procedure to the rows of $\mathbf{F}^{[N-1]}$. Namely, subtract the first row of $\mathbf{F}^{[N-1]}$ from the succeeding $N-1$ rows of $\mathbf{F}^{[N-1]}$ and call the resulting matrix $\widetilde{\mathbf{F}}^{[N-1]}$, multiply the $k$ th row of $\widetilde{\mathbf{F}}^{[n-1]}$ by $\frac{1}{u_{k}-u_{1}}$ for $k=2, \ldots, N$ and call the result $\mathbf{F}^{[1, N-1]}$. Note that

$$
\operatorname{det} \mathbf{F}^{[N-1]}=\operatorname{det} \widetilde{\mathbf{F}}^{[N-1]}
$$

and

$$
\widetilde{\mathbf{F}}^{[N-1]}=\operatorname{diag}\left(1, u_{2}-u_{1}, \ldots, u_{N}-u_{1}\right) \mathbf{F}^{[1, N-1]},
$$


so that

$$
\operatorname{det} \mathbf{F}^{[N-1]}=\left\{\prod_{k=2}^{N}\left(u_{k}-u_{1}\right)\right\} \operatorname{det} \mathbf{F}^{[1, N-1]} .
$$

Continue in this way to get $\mathbf{F}^{[2, N-1]}, \ldots, \mathbf{F}^{[N-1, N-1]}$ where

$$
\operatorname{det} \mathbf{F}^{[n-1, N-1]}=\left\{\prod_{k=n+1}^{N}\left(v_{k}-v_{n}\right)\right\} \operatorname{det} \mathbf{F}^{[n, N-1]}
$$

for $n=1, \ldots, N-1$. The $k$ th row of $\mathbf{F}^{[N-1, N-1]}$ consists of divided differences of order $k-1$ of the first $k$ rows of $\mathbf{F}^{[N-1]}$ with respect to $u_{1}, \ldots, u_{k}$.

Clearly $\mathbf{F}^{[N-1, N-1]}=\left(F\left(\left[U_{k}\right],\left[V_{l}\right]\right)\right)$, and relations (47) through (51) imply that

$$
\operatorname{det}\left(F\left(u_{k}, v_{l}\right)\right)=\left\{\prod_{k>l}\left(u_{k}-u_{l}\right)\left(v_{k}-v_{l}\right)\right\} \operatorname{det}\left(\left(F\left(\left[U_{k}\right],\left[V_{l}\right]\right)\right),\right.
$$

which is the desired result.

Suppose in the $N \times N$ matrix $\left(F\left(u_{k}, v_{l}\right)\right)$ we replace the $p$ th column by

$$
\left(\begin{array}{c}
G\left(u_{1}\right) \\
\vdots \\
G\left(u_{N}\right)
\end{array}\right)
$$

and call the result $\mathbf{F}(U, V ; G(U), p)$. Then a procedure similar to that used in the proof of Theorem 3 allows us to obtain a formula for the determinant of $\mathbf{F}(U, V ; G(U), p)$ as a product involving $\left(u_{k}-u_{l}\right),\left(v_{k}-v_{l}\right)$, and a determinant containing divided differences of $F(u, v)$ and $G(u)$. Since we will use the formula in what follows, we will record it in the case $p=1$.

\section{Theorem 4.}

$$
\operatorname{det} \mathbf{F}(U, V ; G(U), 1)=\prod_{k>l}\left(u_{k}-u_{l}\right) \prod_{k>l>1}\left(v_{k}-v_{l}\right) \sum_{n=1}^{N} G\left(\left[U_{n}\right]\right) S_{n}
$$

where

(i) $U_{k}=\left\{u_{1}, \ldots, u_{k}\right\}$,

(ii) $S_{n}$ is a sum of terms of the form

$$
\pm \prod_{j=1}^{N-1} F\left(\left[U_{k_{j}}\right],\left[V_{l_{j}}\right]\right)
$$

(iii) $k_{1}, \ldots, k_{N-1}$ is a permutation of the terms $\{1, \ldots, N\} \backslash\{n\}$, and

(iv) $V_{l_{j}}=\left\{v_{2}, \ldots, v_{l_{j}}\right\}$ where $l_{1}, \ldots, l_{N-1}$ is a permutation of the set $\{2, \ldots, N\}$.

Note that $G\left(\left[U_{n}\right]\right)$ is a divided difference of $G(u)$ of order $n-1$, while $F\left(\left[U_{k_{j}}\right]\right.$, $\left.\left[V_{l_{j}}\right]\right)$ is a mixed divided difference of $F(u, v)$ of order $k_{j}-1$ in $u$ and of order $l_{j}-2$ in $v$. Also note that the second product on the right-hand side of (52) contains exactly $N(N-1) / 2-(N-1)=(N-1)(N-2) / 2$ terms; so in the case when $N=2$ we take this product to be equal to 1 .

The formulas in the cases $p=2, \ldots, N$ are, of course, completely analogous. 
6.

We use the notation established in the previous sections.

Recall that $m_{k}(\epsilon)$ denotes the $k$ th moment of $\phi_{\epsilon}(x)$, see (7). The analogues of properties (17) and (18) in the general case are

$$
0<r_{0} \leq \frac{\operatorname{det}\left(m_{k+l-2}(\epsilon)\right)}{m_{2(N-1)}(\epsilon)^{N / 2}} \leq r_{1}<\infty
$$

where $r_{0} \leq r_{1}$ are constants which are independent of $\epsilon$ and

$$
\lim _{\epsilon \rightarrow 0} \frac{\int_{-\infty}^{\infty} \omega(x) x^{2(N-1)} \phi_{\epsilon}(x) d x}{m_{2(N-1)}(\epsilon)}=0 .
$$

We recall, see Section 2, that these properties are quite natural and are enjoyed by a large class of approximations of the identity. For more examples, see Section 8.

For simplicity in what follows, we will often use the symbol $\asymp$ to denote the relationship between $\operatorname{det}\left(m_{k+l-2}(\epsilon)\right)$ and $m_{2(N-1)}(\epsilon)^{N / 2} \operatorname{described}$ by (54). Thus, for example, we may write $\operatorname{det}\left(m_{k+l-2}(\epsilon)\right) \asymp m_{2(N-1)}(\epsilon)^{N / 2}$ as an abbreviation for (54).

Theorem 5. If $\phi_{\epsilon}$ enjoys properties (54) and (55), then

$$
\operatorname{det}\left(\Phi\left(u_{k}-v_{l}\right)\right)=\left\{\prod_{k>l}\left(u_{k}-u_{l}\right)\left(v_{k}-v_{l}\right)\right\} c\left\{\operatorname{det}\left(\Phi^{(k+l-2)}(0)\right)+S\right\}
$$

where

$$
\frac{1}{c}=(-1)^{N(N-1) / 2} \prod_{k=0}^{N-1}(k !)^{2}
$$

and $S$, which depends on $U=\left\{u_{1}, \ldots, u_{N}\right\}, V=\left\{v_{1}, \ldots, v_{N}\right\}$, and $\phi_{\epsilon}$, satisfies

$$
S=o\left(\left\{m_{2(N-1)}(\epsilon)\right\}^{N / 2}\right) \text { as } \epsilon \rightarrow 0 .
$$

Proof. Application of Theorem 3 with $F(u, v)=\Phi(u-v)$ and the use of identity (45) to re-express the divided differences as derivatives result in

$$
\operatorname{det}\left(\Phi\left(u_{k}-v_{l}\right)\right)=\left\{\prod_{k>l}\left(u_{k}-u_{l}\right)\left(v_{k}-v_{l}\right)\right\} c \operatorname{det}\left(\Phi^{(k+l-2)}\left(z_{k, l}\right)\right)
$$

where the constant $c$ is given by (57) and the $z_{k, l}$ denote appropriately chosen pairs of real numbers; see Remark 3.1 and the remark immediately succeeding identity (45). Now, if we write

$$
\Phi^{(n)}(z)=\Phi^{(n)}(0)+\Omega_{n}(z)
$$

where

$$
\Omega_{n}(z)=\Phi^{(n)}(z)-\Phi^{(n)}(0)
$$

the last determinant may be re-expressed as

$$
\operatorname{det}\left(\Phi^{(k+l-2)}\left(z_{k, l}\right)\right)=\operatorname{det}\left(\Phi^{(k+l-2)}(0)\right)+S
$$

where $S$ is a finite sum of terms of the form

$$
T=\prod_{j=1}^{n} \Omega_{k_{j}+l_{j}-2}\left(z_{k_{j}, l_{j}}\right) \prod_{j=n+1}^{N} \Phi^{\left(k_{j}+l_{j}-2\right)}(0)
$$


where $k_{1}, \ldots, k_{N}$ and $l_{1}, \ldots, l_{N}$ are permutations of $1, \ldots, N$. Note that in the last identity (i) the integer parameter $n$ satisfies $1 \leq n \leq N$; (ii) the second product might contain no terms, in which case it should be interpreted simply as 1 ; and (iii) the first product always contains at least one non-trivial term.

To complete the proof it suffices to show that

$$
\lim _{\epsilon \rightarrow 0} \frac{T}{\left\{m_{2(N-1)}(\epsilon)\right\}^{N / 2}}=0 .
$$

To this end, first consider the second product on the right-hand side of identity (59), and write

$$
\left|\prod_{j=n+1}^{N} \Phi^{\left(k_{j}+l_{j}-2\right)}(0)\right| \leq \prod_{j=n+1}^{N} \int_{-\infty}^{\infty}|x|^{k_{j}+l_{j}-2} \phi_{\epsilon}(x) d x \leq\left\{\int_{-\infty}^{\infty} x^{2(N-1)} \phi_{\epsilon}(x) d x\right\}^{p_{2}}
$$

where $p_{2}=\left\{\sum_{j=n+1}^{N}\left(k_{j}+l_{j}-2\right)\right\} /\{2(N-1)\}$.

Next, note that at most one of the terms in the first product can have $k_{j}+l_{j}=$ $2 N$. If none of the terms have this property, let

$$
\omega_{z}(x)=\left|e^{i z x}-1\right|
$$

and write

$$
\begin{aligned}
\left|\prod_{j=1}^{n} \Omega_{k_{j}+l_{j}-1}\left(z_{k_{j}, l_{j}}\right)\right| & \leq \prod_{j=1}^{n} \int_{-\infty}^{\infty} \omega_{z_{k_{j}, l_{j}}}(x)|x|^{k_{j}+l_{j}-2} \phi_{\epsilon}(x) d x \\
& \leq\left\{\prod_{j=1}^{n}\left(\int_{-\infty}^{\infty} \omega_{z_{k_{j}, l_{j}}}^{q_{j}}(x) \phi_{\epsilon}(x) d x\right)^{1 / q_{j}}\right\}\left\{m_{2(N-1)}(\epsilon)\right\}^{p_{1}}
\end{aligned}
$$

where

$$
p_{1}=\left\{\sum_{j=1}^{n}\left(k_{j}+l_{j}-2\right)\right\} /\{2(N-1)\}
$$

and

$$
1 / q_{j}=1-\left\{k_{j}+l_{j}-2\right\} /\{2(N-1)\} .
$$

If one of the terms has this property, without loss of generality we may take $k_{1}+l_{1}=2 N$ and compute as above to get

$$
\begin{aligned}
& \left|\prod_{j=1}^{n} \Omega_{k_{j}+l_{j}-2}\left(z_{k_{j}, l_{j}}\right)\right| \\
& \leq \int_{-\infty}^{\infty} \omega_{z_{N, N}}(x) x^{2(N-1)} \phi_{\epsilon}(x) d x \\
& \quad \times\left\{\prod_{j=2}^{n}\left(\int_{-\infty}^{\infty} \omega_{z_{k_{j}, l_{j}}}^{q_{j}}(x) \phi_{\epsilon}(x) d x\right)^{1 / q_{j}}\right\}\left\{m_{2(N-1)}(\epsilon)\right\}^{p_{1}}
\end{aligned}
$$

where $p_{1}=\left\{\sum_{j=2}^{n}\left(k_{j}+l_{j}-2\right)\right\} /\{2(N-1)\}$ and the $q_{j}$ are as above. 
It follows that if one of the terms $k_{j}+l_{j}=2 N$, then

$$
\begin{aligned}
|T| \leq & \int_{-\infty}^{\infty} \omega_{z_{N, N}}(x) x^{2(N-1)} \phi_{\epsilon}(x) d x \\
& \times\left\{\prod_{j=2}^{n}\left(\int_{-\infty}^{\infty} \omega_{z_{k_{j}, l_{j}}}^{q_{j}}(x) \phi_{\epsilon}(x) d x\right)^{1 / q_{j}}\right\}\left\{m_{2(N-1)}(\epsilon)\right\}^{N / 2-1}
\end{aligned}
$$

otherwise

$$
|T| \leq\left\{\prod_{j=1}^{n}\left(\int_{-\infty}^{\infty} \omega_{z_{k_{j}, l_{j}}}^{q_{j}}(x) \phi_{\epsilon}(x) d x\right)^{1 / q_{j}}\right\}\left\{m_{2(N-1)}(\epsilon)\right\}^{N / 2},
$$

where $1 / q_{j}=1-\left\{k_{j}+l_{j}-2\right\} /\{2(N-1)\}$. Either case implies identity (60).

We can now determine the limits considered at the beginning of Section 5. Recall the notation agreed to there, particularly the meaning of $N, P_{f, \epsilon}(x)$ and $P_{f, 0}(x)$.

Corollary 1. If $f(x)=e^{-i \alpha x}$ for some real number $\alpha$ and $\phi_{\epsilon}$ enjoys properties (54) and (55), then

$$
\lim _{\epsilon \rightarrow 0} P_{f, \epsilon}(x)=P_{f, 0}(x)
$$

Proof. If

$$
P_{f, \epsilon}=\sum_{k=1}^{N} c_{\epsilon, k} e^{-i \nu_{k} x}
$$

then for $\epsilon>0$,

$$
c_{\epsilon, k}=\frac{\operatorname{det} \mathbf{F}(U, V ; G(U), k)}{\operatorname{det} \mathbf{F}(U, V)}
$$

where $F(u, v)=\Phi(u-v), G(u)=\Phi(u-\alpha)$, and $U=V=\left\{\nu_{1}, \ldots, \nu_{N}\right\}$. Note that $\mathbf{F}(U, V ; G(U), k)$ is $\left(\Phi\left(\nu_{k}-\nu_{l}\right)\right)$ with the $k$ th column replaced with

$$
\left(\begin{array}{c}
\Phi\left(\nu_{1}-\alpha\right) \\
\vdots \\
\Phi\left(\nu_{N}-\alpha\right)
\end{array}\right)
$$

The formula for $c_{0, k}$, see (39), may be simplified to

$$
c_{0, k}=\prod_{\{j: j \neq k\}} \frac{\nu_{j}-\alpha}{\nu_{j}-\nu_{k}} .
$$

To see that

$$
\lim _{\epsilon \rightarrow 0} c_{\epsilon, k}=c_{0 . k},
$$

use Theorem (5) and the abbreviation $W=\operatorname{det}\left(\Phi^{(k+l-2)}(0)\right)$ to re-express $c_{\epsilon, k}$ as

$$
c_{\epsilon, k}=\frac{\left\{\prod_{k>l}\left(\mu_{k}-\mu_{l}\right)\left(\nu_{k}-\nu_{l}\right)\right\} c\left\{1+\frac{S 1}{W}\right\}}{\left\{\prod_{k>l}\left(\nu_{k}-\nu_{l}\right)^{2}\right\} c\left\{1+\frac{S 2}{W}\right\}}
$$

where $\mu_{k}=\alpha$ and $\mu_{j}=\nu_{j}$ for $j \in\{1, \ldots, N\} \backslash\{k\}$. S2 depends on the parameters $\nu_{1}, \ldots, \nu_{N}$ and $\epsilon$, while, in addition to these parameters, $S 1$ also depends on $\alpha$. In both cases, $k=1$ and 2 ,

$$
S k=o\left(\left\{m_{2(N-1)}(\epsilon)\right\}^{N / 2}\right) \text { as } \epsilon \rightarrow 0 .
$$


This behavior of $S 1$ and $S 2$, together with the fact that $W$ is a constant multiple of $\operatorname{det}\left(m_{k+l-2}(\epsilon)\right)$ and thus

$$
W=W(\epsilon) \asymp\left\{m_{2(N-1)}(\epsilon)\right\}^{N / 2},
$$

allows us to deduce (62) from the last expressions for $c_{\epsilon, k}$ and $c_{0, k}$.

The identity (62) is, of course, equivalent to (61).

In view of the above corollary and the fact that $P_{f, \epsilon}(x)$ is linear in $f$, it is tempting to use the representation

$$
f(x)=\frac{1}{2 \pi} \int_{-\infty}^{\infty} e^{i \xi x} \hat{f}(\xi) d \xi
$$

to conclude that (61) is valid for all sufficiently well-behaved $f$. However, to avoid the usual difficulties presented by the interchange of limiting procedures, we base our conclusion on the following theorem.

Theorem 6. Suppose $f$ is a bounded measurable function on $\mathbb{R}$ which is $N-1$ times continuously differentiable in a neighborhood of the origin and $f(0)=f^{\prime}(0)=\cdots=$ $f^{(N-1)}(0)=0$. If $\phi_{\epsilon}$ enjoys properties (54) and (55), then

$$
\lim _{\epsilon \rightarrow 0} P_{f, \epsilon}(x)=0
$$

for all $x$.

Proof. As in the proof of the corollary, we have

$$
P_{f, \epsilon}=\sum_{k=1}^{N} c_{\epsilon, k} e^{-i \nu_{k} x}
$$

and for $\epsilon>0$,

$$
c_{\epsilon, k}=\frac{\operatorname{det} \mathbf{F}(U, V ; G(U), k)}{\operatorname{det}\left(\Phi\left(u_{j}-v_{k}\right)\right)}
$$

where $F(u, v)=\Phi(u-v)$ and $U=V=\left\{\nu_{1}, \ldots, \nu_{N}\right\}$; but here

$$
G(u)=\int_{-\infty}^{\infty} f(x) e^{i u x} \phi_{\epsilon}(x) d x .
$$

To prove the theorem, it suffices to show that

$$
\lim _{\epsilon \rightarrow 0} c_{\epsilon, 1}=0,
$$

since the validity of the result for values of the subscript $k$ other than one follows by permutation of indices.

To verify (64), use Theorem 4 to write

$$
\operatorname{det} \mathbf{F}(U, V ; G(U), 1)=\prod_{k>l}\left(u_{k}-u_{l}\right) \prod_{k>l>1}\left(v_{k}-v_{l}\right) \sum_{n=1}^{N} G^{(n-1)}\left(z_{n}\right) S_{n}
$$

where $S_{n}$ is a sum of terms of the form

$$
\pm \prod_{j=1}^{N-1} \frac{\Phi^{\left(k_{j}-l_{j}-3\right)}\left(z_{k_{j}, l_{j}}\right)}{\left(k_{j}-1\right) !\left(l_{j}-2\right) !} .
$$

Here we used (45) and the remark which immediately succeeds it, to re-express the divided differences in formulas (52) and (53) as derivatives. Also note that here (i) 
$U_{k}=\left\{\nu_{1}, \ldots, \nu_{k}\right\}$, (ii) $k_{1}, \ldots, k_{N-1}$ is a permutation of the terms $\{1, \ldots, N\} \backslash\{n\}$, (iii) $V_{l_{j}}=\left\{\nu_{2}, \ldots, \nu_{l_{j}}\right\}$, where $l_{1}, \ldots, l_{N-1}$ is a permutation of the terms $\{2, \ldots, N\}$, and (iv) $z_{n}$ and $z_{k_{j}, l_{j}}$, for $n=1, \ldots, N$ and $j=1, \ldots, N-1$, denote appropriately chosen pairs of real numbers, see Remark 3.1.

Since $\operatorname{det}\left(\Phi\left(\nu_{k}-\nu_{l}\right)\right) \asymp\left\{m_{2(N-1)}(\epsilon)\right\}^{N / 2}$, to see (64) it suffices to show that

$$
G^{(n-1)}\left(z_{n}\right) \prod_{j=1}^{N-1} \Phi^{\left(k_{j}-l_{j}-3\right)}\left(z_{k_{j}, l_{j}}\right)=o\left(\left\{m_{2(N-1)}(\epsilon)\right\}^{N / 2}\right) \text { as } \epsilon \rightarrow 0
$$

for $n=1, \ldots, N$.

To see (65), first note that

$$
G^{(n-1)}\left(z_{n}\right)=\int_{-\infty}^{\infty}(i x)^{n-1} f(x) e^{i z_{n} x} \phi_{\epsilon}(x) d x
$$

and $|f(x)| \leq \omega(x)|x|^{N-1}$ where $\omega(x)$ is a bounded continuous function such that $\omega(0)=0$. Indeed,

$$
\omega(x) \leq\left|\operatorname{Re} f^{(N-1)}\left(x_{1}\right)+i \operatorname{Im} f^{(N-1)}\left(x_{2}\right)\right| /(N-1) !
$$

for some $x_{1}$ and $x_{2}$ between 0 and $x$ when $x$ is in a sufficiently small neighborhood of the origin. Thus

$$
\left|G^{(n-1)}\left(z_{n}\right)\right| \leq \int_{-\infty}^{\infty} \omega(x)|x|^{N+n-2} \phi_{\epsilon}(x) d x
$$

which, if $n=1, \ldots N-1$, is

$$
\leq\left\{\int_{-\infty}^{\infty} \omega(x)^{p} \phi_{\epsilon}(x) d x\right\}^{1 / p}\left\{\int_{-\infty}^{\infty}|x|^{2(N-1)} \phi_{\epsilon}(x) d x\right\}^{1 / q}
$$

where

$$
\frac{1}{p}+\frac{1}{q}=1 \text { and } \frac{1}{q}=\frac{N+n-2}{2(N-1)}
$$

Next, using an argument similar to that used in the proof of Theorem note that

$$
\left|\prod_{j=1}^{N-1} \Phi^{\left(k_{j}-l_{j}-3\right)}\left(z_{k_{j}, l_{j}}\right)\right| \leq \prod_{j=1}^{N-1} \int_{-\infty}^{\infty}|x|^{k_{j}+l_{j}-3} \phi_{\epsilon}(x) d x \leq\left\{m_{2(N-1)}(\epsilon)\right\}^{r /(2 N-2)}
$$

where

$$
r=\sum_{j=1}^{N-1}\left\{k_{j}+l_{j}-3\right\}=\left\{\frac{N(N+1)}{2}-n\right\}+\left\{\frac{N(N+1)}{2}-1\right\}-3(N-1)
$$

or, more simply,

$$
r=(N-1)^{2}-n+1 .
$$

Since the exponents in the last two strings of inequalities satisfy

$$
\frac{1}{q}+\frac{r}{2(N-1)}=\frac{N}{2}
$$

it follows that if $n=1, \ldots N-1$, then

$$
\left|G^{(n-1)}\left(z_{n}\right) \prod_{j=1}^{N-1} \Phi^{\left(k_{j}-l_{j}-3\right)}\left(z_{k_{j}, l_{j}}\right)\right| \leq \int_{-\infty}^{\infty}\left\{\omega(x)^{p} \phi_{\epsilon}(x) d x\right\}^{1 / p}\left\{m_{2(N-1)}(\epsilon)\right\}^{N / 2},
$$


and if $n=N$, then

$$
\begin{aligned}
& \left|G^{(n-1)}\left(z_{n}\right) \prod_{j=1}^{N-1} \Phi^{\left(k_{j}-l_{j}-3\right)}\left(z_{k_{j}, l_{j}}\right)\right| \\
& \quad \leq \int_{-\infty}^{\infty} \omega(x)|x|^{2(N-1)} \phi_{\epsilon}(x) d x\left\{m_{2(N-1)}(\epsilon)\right\}^{N / 2-1} .
\end{aligned}
$$

Either case implies [65].

The following statement, which is the main result in this section, is an immediate consequence of Theorem [ 6 and the facts that (i) the mapping $f \rightarrow P_{f, \epsilon}$ is linear in $f$ and (ii) if $f=\sum_{k=1}^{N} c_{k} e^{-i \nu_{k} x}$, then $P_{f, \epsilon}=f$.

Theorem 7. Suppose $f$ is a bounded measurable function on $\mathbb{R}$ which is $N-1$ times continuously differentiable in a neighborhood of the origin. If $\phi_{\epsilon}$ enjoys properties (54) and (55), then

$$
\lim _{\epsilon \rightarrow 0} P_{f, \epsilon}(x)=P_{f, 0}(x)
$$

for all $x$.

For the sake of completeness we remark that there is nothing special about the origin. The above results are valid mutatis mutandis with 0 replaced by any fixed point $x_{0}$.

7.

Suppose $\mathcal{N}=\left\{\nu_{1}, \ldots, \nu_{N}\right\}$ is a finite subset of the integer lattice $\mathbb{Z}$ and $S$ is a piecewise polynomial spline of order $2 k$ with knot set $\mathcal{X}=\mathbb{Z} \backslash \mathcal{N}$. We are interested in the behavior of $S$ when $\left.S\right|_{\mathcal{X}}$ remains fixed and the order $2 k$ goes to $\infty$.

As indicated in the introduction, such phenomena have been investigated earlier in the cases when the set of knots is a lattice or even a more general complete interpolating sequence for the classical Paley-Wiener space. $\mathcal{X}$ is not such a sequence when $\mathcal{N} \neq \emptyset$.

Specifically, we are interested in $L_{n, 2 k}(x)$, indexed by $n$ in $\mathcal{X}$, the uniquely defined splines of order $2 k$ of no greater than polynomial growth which satisfy

$$
L_{n, 2 k}(m)= \begin{cases}1 & \text { if } m=n, \\ 0 & \text { if } m \in \mathcal{X} \backslash\{n\} .\end{cases}
$$

$L_{n, 2 k}$ may be succinctly described as the tempered distribution which satisfies

$$
\frac{d^{2 k}}{d x^{2 k}} L_{n, 2 k}(x)=\sum_{l \in \mathcal{X}} a_{l} \delta(x-l)
$$

and enjoys property (67). In the above expression, $\delta(x)$ represents the unit Dirac measure at the origin. The fact that $L_{n, 2 k}$ exists and is unique is well known; for examples, see [1].

Past work shows that it may be useful to consider the Fourier transform $\hat{L}_{n, 2 k}(\xi)$ of $L_{n, 2 k}(x)$. The normalization of the Fourier transform used here gives

$$
\hat{f}(\xi)=\int_{-\infty}^{\infty} f(x) e^{-i \xi x} d x
$$


as the Fourier transform of an integrable function $f$. For the basic properties of Fourier transforms and tempered distributions, see, for example, [7].

\section{Proposition 1.}

$$
\hat{L}_{n, 2 k}(\xi)=\left\{e^{-i n \xi}-P_{n, k}(\xi)\right\} \frac{|\xi|^{-2 k}}{\sum_{j=-\infty}^{\infty}|\xi-2 \pi j|^{-2 k}}
$$

where

$$
P_{n, k}(\xi)=\sum_{\nu_{j} \in \mathcal{N}} c_{j} e^{-i \nu_{j} \xi}
$$

satisfies

$$
\int_{0}^{2 \pi} \frac{e^{-i n \xi}-P_{n, k}(\xi)}{\sum_{j=-\infty}^{\infty}|\xi-2 \pi j|^{-2 k}} e^{i \nu_{j} \xi} d x=0 \text { for } \nu_{j} \in \mathcal{N} .
$$

Proof. The properties of $L_{n, 2 k}(x)$ imply that its Fourier transform is a distribution of the form

$$
\hat{L}_{n, 2 k}(\xi)=P(\xi)|\xi|^{-2 k}
$$

where $P(\xi)$ is a smooth $2 \pi$ periodic function which satisfies both

$$
\int_{0}^{2 \pi} P(\xi) e^{i \nu \xi} d \xi=0 \text { for } \nu \in \mathcal{N}
$$

and

$$
\frac{1}{2 \pi} \int_{-\infty}^{\infty} P(\xi)|\xi|^{-2 k} e^{i m \xi} d \xi= \begin{cases}1 & \text { if } m=n, \\ 0 & \text { if } m \in \mathcal{X} \backslash\{n\} .\end{cases}
$$

Since the left-hand side of the last identity can be re-expressed as

$$
\frac{1}{2 \pi} \int_{0}^{2 \pi} P(\xi)\left\{\sum_{j=-\infty}^{\infty}|\xi-2 \pi j|^{-2 k}\right\} e^{i m \xi} d \xi,
$$

these identities imply

$$
P(\xi) \sum_{j=-\infty}^{\infty}|\xi-2 \pi j|^{-2 k}=e^{-i n \xi}-\sum_{\nu_{j} \in \mathcal{N}} c_{j} e^{-i \nu_{j} \xi},
$$

and the conclusion of the proposition follows.

Let

$$
\phi_{\epsilon}(\xi-\pi)=c_{\epsilon} \chi(\xi-\pi) /\left\{\sum_{j=-\infty}^{\infty}|\xi-2 \pi j|^{-1 / \epsilon}\right\}
$$

where $0<\epsilon<1, \chi(x)$ is the indicator function of the interval $[-\pi, \pi]$, and $c_{\epsilon}$ is a constant chosen so that $\int_{-\infty}^{\infty} \phi_{\epsilon}(x) d x=1$. It should be clear that relation (70) is equivalent to

$$
\begin{aligned}
& \int_{-\infty}^{\infty} P_{n, k}(\xi) e^{i \nu_{j} \xi} \phi_{\epsilon}(\xi) d \xi=\int_{-\infty}^{\infty} e^{-i n \xi} e^{i \nu_{j} \xi} \phi_{\epsilon}(\xi) d \xi \\
& \text { for } \nu_{j} \in \mathcal{N}, \epsilon=1 /(2 k), \text { and } k=1,2, \ldots .
\end{aligned}
$$

Hence if $\phi_{\epsilon}(\xi), \epsilon=1 /(2 k)$, is an approximation of the identity which enjoys properties (54) and (55), then in view of the results in the previous section the nature 
of $P_{n, k}(\xi)$ as $k \rightarrow \infty$ should be clear. Indeed, in the next section we show the following:

Proposition 2. The function $\phi_{\epsilon}(\xi)$ defined by (71), with $\epsilon>0$, is an approximation of the identity as $\epsilon \rightarrow 0$ which for every integer $N$ enjoys properties (54) and (55)).

As a corollary of this we have the following result concerning the nature of $L_{n, 2 k}(x)$ as $k \rightarrow \infty$.

\section{Theorem 8.}

$$
\lim _{k \rightarrow \infty} L_{n, 2 k}(x)=(-1)^{n} \frac{\sin \pi x}{\pi(x-n)} \prod_{\nu_{j} \in \mathcal{N}} \frac{n-\nu_{j}}{x-\nu_{j}}
$$

uniformly in $x \in \mathbb{R}$.

Proof. Let $\chi(\xi)$ be the indicator function of the interval $[-\pi, \pi]$, and note that

$$
\lim _{k \rightarrow \infty} \frac{|\xi|^{-2 k}}{\sum_{j=-\infty}^{\infty}|\xi-2 \pi j|^{-2 k}}=\chi(\xi)
$$

in $L^{1}(\mathbb{R})$. In view of $(72)$, Proposition 2 Theorem 7 and the remark immediately succeeding the statement of Theorem 7

$$
\lim _{k \rightarrow \infty} P_{n, k}(\xi)=P_{n}(\xi)
$$

where $P_{n}(x)$ is the unique trigonometric polynomial of the form (69) which satisfies

$$
P_{n}^{(l)}(\pi)=\left.\frac{d^{l}}{d x^{l}} e^{-i n \xi}\right|_{\xi=\pi}=(-i n)^{l}(-1)^{n} \text { for } l=0, \ldots, N-1 .
$$

We may conclude that $\hat{L}_{n, 2 k}(\xi)$ converges to

$$
\hat{L}_{n}(\xi)=\left\{e^{-i n \xi}-P_{n}(\xi)\right\} \chi(\xi)
$$

in $L^{1}(\mathbb{R})$, and hence $L_{n, 2 k}(x)$ converges uniformly to $L_{n}(x)$ as $k \rightarrow \infty$, where

$$
L_{n}(x)=\frac{\sin \pi x}{\pi}\left\{\frac{(-1)^{n}}{x-n}-\sum_{\nu_{j} \in \mathcal{N}} \frac{(-1)^{\nu_{j}} c_{j}}{x-\nu_{j}}\right\}
$$

and the $c_{j}$ are the coefficients of $P_{n}(\xi)$. The last identity may be re-expressed as

$$
L_{n}(x)=\frac{\sin \pi x}{\pi(x-n)} \frac{Q(x)}{\prod_{\nu_{j} \in \mathcal{N}}\left(x-\nu_{j}\right)}
$$

where $Q$ is a polynomial of degree no greater than $N$. That $Q$ is a constant follows from the fact that $|x|^{N} L_{n}(x)$ is square integrable, because (74) implies the square integrability of $\hat{L}_{n}^{(l)}(\xi), l=0, \ldots, N$. That this constant has the desired value follows from the fact that $L_{n}(n)=1$.

8.

In Section 2 we described a wide class of approximations of the identity which enjoy properties (54) and (55). The objective of this section is to extend this class and, in the process, provide a proof for Proposition 2 
To this end, consider the $\phi_{\epsilon}$ defined by (71). Namely,

$$
\phi_{\epsilon}(x-\pi)=c_{\epsilon} \chi(x-\pi) /\left\{\sum_{j=-\infty}^{\infty}|x-2 \pi j|^{-1 / \epsilon}\right\},
$$

where $0<\epsilon<1, \chi(x)$ is the indicator function of the interval $[-\pi, \pi]$, and $c_{\epsilon}$ is a constant chosen so that $\int_{-\infty}^{\infty} \phi_{\epsilon}(x) d x=1$.

Clearly $\phi_{\epsilon}(x)$ is a non-negative, even function of $x$ with support in the interval $[-\pi, \pi]$. To see that $\phi_{\epsilon}(x)$ is indeed an approximation of the identity, it suffices to show that

$$
\lim _{\epsilon \rightarrow 0} \int_{0}^{\pi-\delta} \phi_{\epsilon}(x-\pi) d x=0 \text { for all } 0<\delta<\pi .
$$

To see (76), for simplicity set $q=1 / \epsilon$ and observe that

$$
1 /\left\{\sum_{j=-\infty}^{\infty}|x-2 \pi j|^{-q}\right\}=|x|^{q} /\left\{1+\sum_{j \in \mathbb{Z} \backslash\{0\}}\{|x| /|x-2 \pi j|\}^{q}\right\},
$$

from which it should be clear that for $0 \leq x \leq \pi$,

$$
\phi_{1 / q}(x-\pi)=\frac{q+1}{\pi^{q+1}} x^{q} b_{q}(x)
$$

where $0<r_{0} \leq b_{q}(x) \leq r_{1}<\infty$ with both $r_{0}$ and $r_{1}$ independent of $q$ and $x$. Hence

$$
\int_{0}^{\pi-\delta} \phi_{1 / q}(x-\pi) d x \leq r_{1} \frac{q+1}{\pi^{q+1}} \int_{0}^{\pi-\delta} x^{q} d x=r_{1}\left\{\frac{\pi-\delta}{\pi}\right\}^{q}
$$

which implies the desired limit (76).

To verify that the $\phi_{\epsilon}(x)$ of (75) also enjoys properties (54) and (55), it may be illuminating to consider a wider class of which (75) is a member.

Proposition 3. Suppose $\phi_{\epsilon}(x)=b_{\epsilon}(x) \psi_{\epsilon}(x)$ where both $b_{\epsilon}(x)$ and $\psi_{\epsilon}(x)$ are nonnegative and satisfy

(i) $0<r_{0} \leq b_{\epsilon}(x) \leq r_{1}<\infty$ with both $r_{0}$ and $r_{1}$ independent of $\epsilon$ and $x$;

(ii) for some positive integer $N$,

$$
0<r_{n, 0} \leq \int_{-\infty}^{\infty}|x|^{n} \epsilon \psi_{\epsilon}(\epsilon x) d x \leq r_{n, 1}<\infty
$$

for $n=0,1, \ldots, 2(N-1)$, where both $r_{n, 0}$ and $r_{n, 1}$ are independent of $\epsilon$;

(iii) for the same $N$ as above

$$
\int_{-\infty}^{\infty}\left|\sum_{k=1}^{N} \xi_{k} x^{k-1}\right|^{2} \epsilon \psi_{\epsilon}(\epsilon x) d x \geq s_{0}\left\{\left|\xi_{1}\right|^{2}+\cdots+\left|\xi_{N}\right|^{2}\right\}
$$

where $s_{0}$ is a positive constant independent of $\epsilon$ and $\xi_{1}, \ldots, \xi_{N}$.

Then

$$
c_{n} r_{0} \epsilon^{n} \leq \int_{-\infty}^{\infty}|x|^{n} \phi_{\epsilon}(x) d x \leq c_{n} r_{1} \epsilon^{n} \text { for } n=0,1, \ldots, 2(N-1),
$$

and if $\mathbf{M}_{N}=\left(m_{k+l-2}\right)$ is the $N \times N$ matrix with coefficients

$$
m_{l+k-2}=\int_{-\infty}^{\infty} x^{k+l-2} \phi_{\epsilon}(x) d x,
$$


where $k$ and $l$ are integers in the range $1, \ldots, N$, then

$$
\left\{r_{0} s_{0}\right\}^{N} \epsilon^{N(N-1)} \leq \operatorname{det} \mathbf{M}_{N} \leq C \epsilon^{N(N-1)}
$$

where $C$ is a constant which depends only on $r_{0,1}, \ldots, r_{2(N-1), 1}$.

Proof. If one writes

$$
\int_{-\infty}^{\infty}|x|^{n} \phi_{\epsilon}(x) d x=\int_{-\infty}^{\infty}|\epsilon x|^{n} b_{\epsilon}(\epsilon x) \epsilon \psi_{\epsilon}(\epsilon x) d x,
$$

it is clear that (77) is an immediate consequence of items (i) and (ii) above. Similarly, if one writes

$$
\operatorname{det}\left(\int_{-\infty}^{\infty} x^{k+l-2} \phi_{\epsilon}(x) d x\right)=\epsilon^{N(N-1)} \operatorname{det}\left(\int_{-\infty}^{\infty} x^{k+l-2} b_{\epsilon}(\epsilon x) \epsilon \psi_{\epsilon}(\epsilon x) d x\right)
$$

and uses (i) and (ii) to estimate the terms in the last determinant, the right-hand inequality in (78) should be clear. The left-hand inequality of (78) is only slightly more opaque.

To see the left-hand inequality of (78), call the terms in the second determinant in identity (79) $b_{k, l}$ and write

$$
\begin{aligned}
\sum_{k, l} b_{k, l} \xi_{k} \bar{\xi}_{l} & =\int_{-\infty}^{\infty}\left|\sum_{k=1}^{N} \xi_{k} x^{k-1}\right|^{2} b_{\epsilon}(\epsilon x) \epsilon \psi_{\epsilon}(\epsilon x) d x \\
& \geq r_{0} \int_{-\infty}^{\infty}\left|\sum_{k=1}^{N} \xi_{k} x^{k-1}\right|^{2} \epsilon \psi_{\epsilon}(\epsilon x) d x \geq r_{0} s_{0}\left\{\left|\xi_{1}\right|^{2}+\cdots+\left|\xi_{N}\right|^{2}\right\}
\end{aligned}
$$

This implies that the smallest eigenvalue of the strictly positive definite matrix $\left(b_{k, l}\right)$ is bounded below by $r_{0} s_{0}$. Since the determinant of an $N \times N$ strictly positive definite matrix is bounded below by the $N$ th power of its smallest eigenvalue, it follows that

$$
\operatorname{det}\left(b_{k, l}\right) \geq\left\{r_{0} s_{0}\right\}^{N}
$$

which, in view of (79), implies the left-hand inequality in (78).

Corollary 2. If $\phi_{\epsilon}(x)$ is an approximation of the identity of the form $\phi_{\epsilon}(x)=$ $b_{\epsilon}(x) \psi_{\epsilon}(x)$, where both $b_{\epsilon}(x)$ and $\psi_{\epsilon}(x)$ satisfy conditions in Proposition (3), then $\phi_{\epsilon}(x)$ enjoys properties (54) and (55)).

Proof of Proposition 2. The argument consists of showing that the hypotheses of Proposition 3 are satisfied.

To see that the $\phi_{\epsilon}$ defined by (75) enjoys the properties of $\phi_{\epsilon}$ in Proposition 3 the changes of variable $x \rightarrow \pi x$ and $q=1 / \epsilon$ imply that it suffices to consider

$$
\phi_{1 / q}(x)=a_{q}(x) q(1-|x|)_{+}^{q}
$$

where $0<r_{0} \leq a_{q}(x) \leq r_{1}<\infty$ with both $r_{0}$ and $r_{1}$ independent of $q$ and $x$. For any real number $u$ the notation $u_{+}$has the usual meaning, namely, $u_{+}=u$ if $u \geq 0$ and $u_{+}=0$ otherwise.

To see that $\psi_{1 / q}(x)=q(1-|x|)_{+}^{q}$ enjoys conditions (ii) and (iii) of the Proposition, write

$$
\int_{-\infty}^{\infty}|x|^{n}\{1 / q\} \psi_{1 / q}(x / q) d x=2 \int_{0}^{q} x^{n}(1-x / q)^{q} d x=2 q^{n+1} \int_{0}^{1} x^{n}(1-x)_{+}^{q} d x
$$


and

$$
\int_{0}^{1} x^{n}(1-x)_{+}^{q} d x=\frac{\Gamma(n+1) \Gamma(q+1)}{\Gamma(n+q+2)}=\frac{n ! \Gamma(q+1)}{\Gamma(q+1)(q+1) \cdots(q+n+1)},
$$

where $\Gamma(z)$ denotes the classical Gamma function. The last two identities imply

$$
\int_{-\infty}^{\infty}|x|^{n}\{1 / q\} \psi_{1 / q}(x / q) d x=2 \frac{n !}{(1+1 / q) \cdots(1+(n+1) / q)},
$$

which allows us to conclude that, for sufficiently large $q$,

$$
\frac{n !}{2^{n}} \leq \int_{-\infty}^{\infty}|x|^{n}\{1 / q\} \psi_{1 / q}(x / q) d x \leq 2 n ! .
$$

Inequalities (81) show that property (ii) of the Proposition is valid for all $n$.

The fact that property (iii) is valid for all $N$ follows from the fact that

$$
\{1 / q\} \psi_{1 / q}(x / q)=(1-|x| / q)_{+}^{q} \geq(1-|x|)_{+}
$$

for all $q>1$. Simply write

$$
\begin{aligned}
\int_{-\infty}^{\infty} & \left|\sum_{k=1}^{N} \xi_{k} x^{k-1}\right|^{2}(1-|x| / q)_{+}^{q} d x \\
& \geq \int_{-\infty}^{\infty}\left|\sum_{k=1}^{N} \xi x^{k-1}\right|^{2}(1-|x|)_{+} d x \geq s_{0}\left\{\left|\xi_{1}\right|^{2}+\cdots+\left|\xi_{N}\right|^{2}\right\} .
\end{aligned}
$$

\section{REFERENCES}

[1] C. deBoor, Odd degree spline interpolation at a bi-infinite knot sequence, in Approximation Theory, R. Schaback and K. Scherer, eds., Lecture Notes in Mathematics, Vol. 556, Springer Verlag, Berlin, 1976, 30-53. MR 58:29610

[2] C. deBoor, K. Höllig, S. Riemenschneider, Convergence of cardinal series. Proc. Amer. Math. Soc. 98 (1986), 457-460. MR 87j:41057

[3] P. J. Davis, Interpolation and Approximation, Dover, New York, 1975. MR 52:1089

[4] M. Golomb, $\mathcal{H}^{m, p}$-extensions by $\mathcal{H}^{m, p}$-splines, J. Approx. Theory, 5, (1972), 238-275. MR 49:937

[5] Yu. Lyubarskii and W. R. Madych, The recovery of irregularly sampled band limited functions via tempered splines, J. Functional Analysis, 155 (1994), 201-222. MR 96d:41013.

[6] I. J. Schoenberg, Cardinal interpolation and spline function VII: The behavior of cardinal spline interpolation as their degree tends to infinity, J. Analyse Math. 27, (1974), 205-229. MR 58:12097b

[7] E. M. Stein and G. Weiss, Introduction to Fourier Analysis on Euclidean Spaces, Princeton Univ. Press, Princeton, N. J., 1971. MR 46:4102

[8] A. Zygmund, Trigonometric Series, 2nd ed., Cambridge Univ. Press, Cambridge, 1968. MR 38:4882

Department of Mathematics, U-9, University of Connecticut, Storrs, Connecticut 06269-3009

E-mail address: madych@uconn.edu 